\title{
Communication : s'adapter à une nouvelle époque, à de nouveaux besoins
}

\author{
par Tania Mysak
}

$\mathrm{L}$

ors d'une rencontre récente, une femme nous a raconté son rétablissement après une blessure à la tête. Une chute en apparence banale lui a occasionné une aphasie suffisamment grave pour compliquer les communications même les plus simples. Sa vie, tant familiale que professionnelle, s'en est trouvée altérée pour toujours. Même si son discours visait à dévoiler l'importance de la " perspective du patient ", il m’a poussée à réfléchir à mes années de pratique, principalement aux cas des patients souffrant de troubles neurologiques. Combien de vies ai-je vues être profondément bouleversées par des blessures minant la compréhension ou la production du langage?

Ces moments nous font réaliser à quel point nous tenons la capacité de communiquer pour acquise jusqu'à ce qu'elle fasse défaut. Toutefois, il nous arrive de ne pas tout comprendre ou d'être incompris même lorsque nos phrases sont correctement construites et distinctement prononcées. Ainsi, il se peut qu'un patient ne saisisse pas entièrement l'information ou les choix que lui communique un prestataire de soin. Dans un autre ordre d'idées, un courriel bien intentionné, mais mal compris, peut conduire à une succession de courriels qui nécessiteront une rencontre pour clarifier les positions. Ou encore, un leader de pharmacie peut ressentir de la frustration de voir des collègues qui ne sont pas au courant des nouvelles politiques néanmoins maintes fois répétées.

L'Association nationale des organismes de réglementation de la pharmacie évalue la compétence communicationnelle des pharmaciens, mais se concentre surtout sur leurs compétences langagières à l'écrit et à l'oral et sur l'empathie démontrée lors d'entrevues avec les patients; des aptitudes nécessaires, mais qui ne sont peut-être pas suffisantes aujourd'hui, étant donné la complexité des défis communicationnels et des différentes façons d'accéder à l'information.

Les cibles du programme Excellence de la Société canadienne des pharmaciens d'hôpitaux (SCPH) visent aussi les compétences communicationnelles. Notre sondage (https://www.cshp.ca/ what-we-heard) révèle qu'à peine deux tiers des patients (68\%) se disaient satisfaits de leur communication avec l'équipe de la pharmacie. Une proportion semblable de patients considérait que la communication avec leurs fournisseurs de soins de santé n'était pas bonne. Si vous réfléchissez à votre pratique ou à la gestion de votre équipe, que faudrait-il faire pour que la communication avec vos patients ou collègues soit efficace? En tant que professionnels, pouvons-nous exprimer clairement notre valeur et nos besoins aux parties prenantes externes? Ou parlons-nous un jargon de pharmacien qui nous empêche de communiquer efficacement à divers auditoires.

Il en est de même à la $\mathrm{SCPH}$, nous devons réfléchir aux échanges avec nos membres et avec les parties prenantes externes. Notre équipe et des volontaires ont consacré de nombreuses heuresà la création de produits de haute qualité : nos activités éducatives, des ressources pour la pratique, le programme Excellence et même ce journal. Nos membres connaissent-ils ces produits? La SCPH a travaillé d'arrache-pied pour mettre en place des plans faisant progresser la profession, mais la communication entre la direction et les membres est-elle assez efficace pour qu'elle sache si ces plans répondent aux besoins des membres? Un regard honnête sur les compétences communicationnelles de la SCPH révèle que nous pouvons nous améliorer.

Les outils de communications modernes sont sophistiqués, puissants et complexes, à l'image de nos médicaments en pharmacie. L'exploration de données, les médias sociaux interactifs et la surveillance avancée sont maintenant choses communes. Mais lesquels choisir? Comment les exploiter? Comment entrent-ils en interaction avec nos membres? Ils nécessitent un ensemble de compétences que nous devons acquérir pour établir une réelle discussion avec nos membres et entre les membres. À cet effet, la direction de la SCPH a mis sur pied une équipe de marketing et de communication qui sera à la hauteur de l'excellence déployée au sein de notre pratique professionnelle et de nos équipes de services commerciaux. La première étape sera la création d'un poste de Directeur du marketing et de la communication qui sera pourvu dès ce printemps. Cette nouvelle position nous fournira les compétences communicationnelles nécessaires à la reconnaissance et à la satisfaction des besoins des membres de la SCPH, de nos partenaires et de la profession.

Comme disait George Bernard Shaw : "Le plus grand problème en communication, c'est d'avoir l'illusion qu'elle a eu lieu. »C'est ce que la SCPH cherche à éviter! Restez à l'affût!

[Traduction par l'éditeur]

Tania Mysak, BSP, Pharm. D., est présidente désignée et agente de liaison de la Société canadienne des pharmaciens d'hôpitaux. 